\title{
Many-body effects in a quasi-one-dimensional electron gas
}

\author{
Sanjeev Kumar, ${ }^{1,3,{ }^{*}}$ Kalarikad J. Thomas, ${ }^{1,3}$ Luke W. Smith, ${ }^{2}$ Michael Pepper, ${ }^{1,3}$ Graham L. Creeth,,${ }^{1,3}$ Ian Farrer, ${ }^{2}$ \\ David Ritchie, ${ }^{2}$ Geraint Jones, ${ }^{2}$ and Jonathan Griffiths ${ }^{2}$ \\ ${ }^{1}$ London Centre for Nanotechnology, 17-19 Gordon Street, London WC1H OAH, United Kingdom \\ ${ }^{2}$ Cavendish Laboratory, J.J. Thomson Avenue, Cambridge CB3 OHE, United Kingdom \\ ${ }^{3}$ Department of Electronic and Electrical Engineering, University College London, Torrington Place, London WC1E 7JE, United Kingdom
}

(Received 28 May 2014; revised manuscript received 4 September 2014; published 6 November 2014)

\begin{abstract}
We have investigated electron transport in a quasi-one dimensional (quasi-1D) electron gas as a function of the confinement potential. At a particular potential configuration, and electron concentration, the ground state of a 1D quantum wire splits into two rows to form an incipient Wigner lattice. It was found that application of a transverse magnetic field can transform a double-row electron configuration into a single row due to magnetic enhancement of the confinement potential. The movements of the energy levels have been monitored under varying conditions of confinement potential and in-plane magnetic field. It is also shown that when the confinement is weak, electron occupation drives a reordering of the levels such that the normal ground state passes through the higher levels. The results show that the levels can be manipulated by utilizing their different dependence on spatial confinement and electron concentration, thus enhancing the understanding of many-body interactions in mesoscopic 1D quantum wires.
\end{abstract}

DOI: 10.1103/PhysRevB.90.201304

PACS number(s): 73.21.Hb, 71.70.Ej, 73.23.Ad, 73.63.Nm

There have been many studies of a strongly confined, noninteracting one-dimensional (1D) electron or hole gas in which the electrostatic confinement is provided by a voltage applied to split or patterned gates [1-4]. In general, the results have been in agreement with the predictions of established theories, such as Luttinger theory and spin-charge separation [5], with the exceptions being the 0.7 anomaly arising from a partial spin polarization [6], and a spin polarization when the momentum degeneracy in the ground 1D subband is lifted by a source-drain voltage [7].

Considerable alteration of the electronic properties can occur with modification of the confinement potential. For example, by inducing a reflection at the channel exit, the system can display Kondo behavior which is additive to the 0.7 anomaly, a many-body effect [8]. Recently it has been found that when the confinement is weak, the ground state conductance is no longer $2 e^{2} / h$ but $4 e^{2} / h$ [9-11]. This transition was attributed to the splitting of electrons occupying the first subband into two separate rows, each with a conductance of $2 e^{2} / h$, these rows being the lowest energy state minimizing the electron-electron interaction as the confining harmonic oscillator potential weakens. Such an "incipient Wigner lattice" was predicted by theories suggesting that the first stage in such a formation was the distortion of the line of electrons into a "zigzag" configuration, which on increasing (decreasing) the repulsion (confinement) resulted in a separation into two rows [12-15]. A similar effect has been calculated for two interacting particles in a square box [16]. Although a zigzag cannot be determined by measurement of conductance behavior, it was possible to ascertain that

\footnotetext{
*sanjeev.kumar@ucl.ac.uk
}

Published by the American Physical Society under the terms of the Creative Commons Attribution 3.0 License. Further distribution of this work must maintain attribution to the author(s) and the published article's title, journal citation, and DOI. the first stage in the breakdown of the spatially quantized ground state was the formation of a "bonding" state, followed by a separation into two rows [10]. In the regime where electron-electron interaction is paramount, a number of $1 \mathrm{D}$ states are predicted, including a ferromagnetic state [17] and various spin phases, which has aroused a considerable interest in the research of interacting fermions in quantum wires, such as Ref. [18].

In this Rapid Communication we present results showing in detail the competing roles of spatial confinement and the electron-electron interaction on 1D quantization. We show how it is possible to alter the plateau sequence by a modification of the higher levels as well as the ground state. A surprising change in the nature of the ground state is shown to occur. In addition, we discuss the effects of a transverse magnetic field which is known to enhance confinement and lead to level depopulation.

The device used in the present work was fabricated from a delta doped GaAs/AlGaAs heterostructure grown using molecular beam epitaxy (MBE), where a twodimensional electron gas (2DEG) formed $300 \mathrm{~nm}$ beneath the surface had a mobility in the dark (light) of $1.2 \times$ $10^{6} \mathrm{~cm}^{2} / \mathrm{V} \mathrm{s}\left(3.5 \times 10^{6} \mathrm{~cm}^{2} / \mathrm{V} \mathrm{s}\right)$ and an electron density of $9.0 \times 10^{10} \mathrm{~cm}^{-2}\left(2.0 \times 10^{11} \mathrm{~cm}^{-2}\right)$. A pair of split gates of length $0.4 \mu \mathrm{m}$ and width $0.7 \mu \mathrm{m}$, and a top gate of length $1 \mu \mathrm{m}$ separated by a $200 \mathrm{~nm}$ thick insulating layer of cross-linked poly(methyl methacrylate) (PMMA) were patterned by a standard lithographic technique [9]. Two-terminal differential conductance $(G)$ measurements were performed using an excitation voltage of $10 \mu \mathrm{V}$ at $73 \mathrm{~Hz}$ in a cryofree dilution refrigerator with an electron temperature of $70 \mathrm{mK}$.

The inset of Fig. 1(a) is a schematic diagram of a typical device used in this work with a pair of split gates and a uniform top gate [19]. Figure 1(a) shows the conductance characteristics of the device as a function of split-gate voltage $\left(V_{\mathrm{sg}}\right)$ for a constant top-gate voltage $\left(V_{\mathrm{tg}}\right)$. Strong (weak) confinement is on the left (right). Weakening the confinement results in the loss of the $2 e^{2} / h$ plateau and $4 e^{2} / h$ becomes 


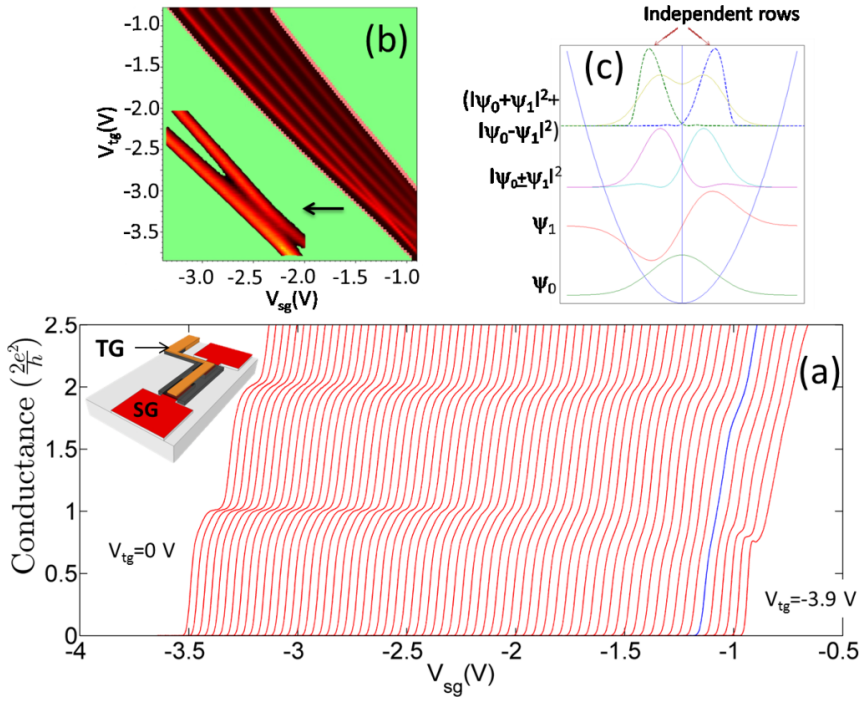

FIG. 1. (Color online) (a) Conductance characteristics of the device for various $V_{\mathrm{tg}}(0$ to $-3.9 \mathrm{~V})$. The inset shows a schematic diagram of a top-gated split-gate device; red shows the split gates (SG), orange is the top gate (TG), and PMMA is shown by a gray layer. (b) Grayscale plot of $d G / d V_{\mathrm{sg}}$ for the data in (a); here the red color indicates the riser in conductance and the dark regions are the conductance plateaus. (c) Schematic diagram showing the hybridization of the ground state and the first excited state wave function in a double-row system. The simple wave functions are shown, whereas in reality they are heavily distorted by the electronelectron interaction.

the ground state, which is indicated by the blue trace at $V_{\mathrm{tg}}=$ $-3.25 \mathrm{~V}$; eventually $2 e^{2} / h$ reappears when the confinement is further weakened. Figure 1(b) is the grayscale plot of transconductance $\left(d G / d V_{\mathrm{sg}}\right)$ as a function of $V_{\mathrm{sg}}$ and $V_{\mathrm{tg}}$ for the data in Fig. 1(a) showing what appears as an anticrossing of the subbands around $V_{\mathrm{tg}}=-3.25 \mathrm{~V}$ corresponding to double-row formation. The inset shows a magnified view of the anticrossing.

The measured conductance depends on the number of occupied subbands below the Fermi level. The first plateau, arising from the ground state, corresponds to a transverse wave function $\Psi_{0}$, termed the 0-state, which figuratively has a halfsine-like characteristic. The second plateau of value $4 e^{2} / h$, resulting from occupation of the second level, is represented by the transverse wave function $\Psi_{1}$, which is sinelike [Fig. 1(c)], and is termed the 1-state. As the confinement is weakened, $\Psi_{0}$ and $\Psi_{1}$ are no longer the simple single-electron wave functions but are modified by the electron-electron interaction. A detailed picture is obtained by considering the grayscale plot [Fig. 1(b)], which shows the variation in the energy levels as the confinement is altered-the left (right) is the strongest (weakest) confinement. A significant feature is the narrowing of the difference between the 0- and 1-states due to more rapid drop of the 1-state, which eventually converges with the 0 -state. We note that the 1-state moves roughly parallel with the higher energy states. One possible explanation of this behavior is the different role of the electron repulsion in the 0 - and 1-states as the confinement weakens. In the 1-state the electrons can describe a highly correlated motion in alternate lobes of the state. The state drops in energy as the confinement width increases, which also reduces the electron-electron repulsion. The 0 -state has the electrons confined near the middle of the channel and does not experience such a reduction in electron repulsion as the 1-state. Hence the 0-state energy only changes due to the weaker confinement, rather than the interaction, and so reduces at a slower rate than the 1-state. When the levels overlap, the result of the mixing is a hybridized wave function which is preferentially located at the edges, corresponding to the formation of two rows, producing a conductance of $4 e^{2} / h$ [schematically shown by the dotted green and blue traces in Fig. 1(c)]. When the confinement potential is further weakened, the 1 -state drops below the 0 -state and the conductance plateau $2 e^{2} / h$ returns, reflecting the reduced role of the interaction in the new ground state.

The device was further characterized by performing dcbias spectroscopy at $V_{\mathrm{tg}}=-3.25 \mathrm{~V}$ where we see the first plateau appearing at $4 e^{2} / h$. Figure 2(a) shows the dc-bias characteristics of the device from 0 to $-3 \mathrm{mV}$. It is observed that there is the usual $0.25\left(2 e^{2} / h\right)$ feature in the strong dc bias, which indicates that either the two rows move together initially or else the source-drain voltage assists one to form before the other. A structure at $0.5\left(2 e^{2} / h\right)$ is also observed; this feature is not observed for strong confinement, which is indicative of the addition of the conductance from two separate rows $[10,11]$. Figure 2(e) is the grayscale plot of the data in Fig. 2(a), where $0.25\left(2 e^{2} / h\right)$ and $0.5\left(2 e^{2} / h\right)$ structures can be traced by looking at the dark, black regions which extend almost parallel to each other as the source-drain bias is increased. In addition, there are half-integer plateaus due to the lifting of the momentum degeneracy which are symmetric about zero source-drain voltage [20].

Figures 2(b)-2(d) show the dc-bias results in the presence of a small transverse magnetic field (perpendicular to the 2D electron gas plane) $B_{\text {tr }}$ of $0.2,0.3$, and $0.45 \mathrm{~T}$, respectively. With an increase in $B_{\mathrm{tr}}$, a feature at $2 e^{2} / h$ starts appearing and at $0.45 \mathrm{~T}$ the first plateau at $2 e^{2} / h$ is completely restored. Such a removal of the direct jump to $4 e^{2} / h$ is due to the additional confinement provided by the magnetic field [2] Broadened plateaus are found at integer multiples of $2 e^{2} / h$ at zero dc bias; on increasing the dc bias, the usual $0.85\left(2 e^{2} / h\right)$ structure appears [21]. A further increase in the dc bias results in the appearance of the $0.25\left(2 e^{2} / h\right)$ structures, and the $0.5\left(2 e^{2} / h\right)$ plateau is much reduced. The grayscale plots of $d G / d V_{\mathrm{sg}}$ for the data in Figs. 2(b)-2(d) are shown in Figs. 2(f)-2(h), respectively, which give further insight into the disappearance of the double row. It may be noticed that with an increase in $B_{\mathrm{tr}}$, the plateaus become sharp and clear due to a reduction in backscattering. Some additional features are observed in Figs. 2(g) and 2(h), such as the splitting of plateaus at zero source-drain bias near $2 e^{2} / h$, which could be due to the approach to the quantum Hall effect [22].

The device was measured again in a different cooldown [23], and the results are shown in Fig. 3(a) for zero magnetic field and Fig. 3(b) with a magnetic field $B_{\|}$of $12 \mathrm{~T}$ applied parallel to the electron gas. Figures 3(c) and 3(d) are the grayscale plots of $d G / d V_{\mathrm{sg}}$ for the data shown in Figs. 3(a) and 3(b), respectively. The most significant feature of Fig. 3(a) is that as the confinement weakens, the 0 -state passes through 

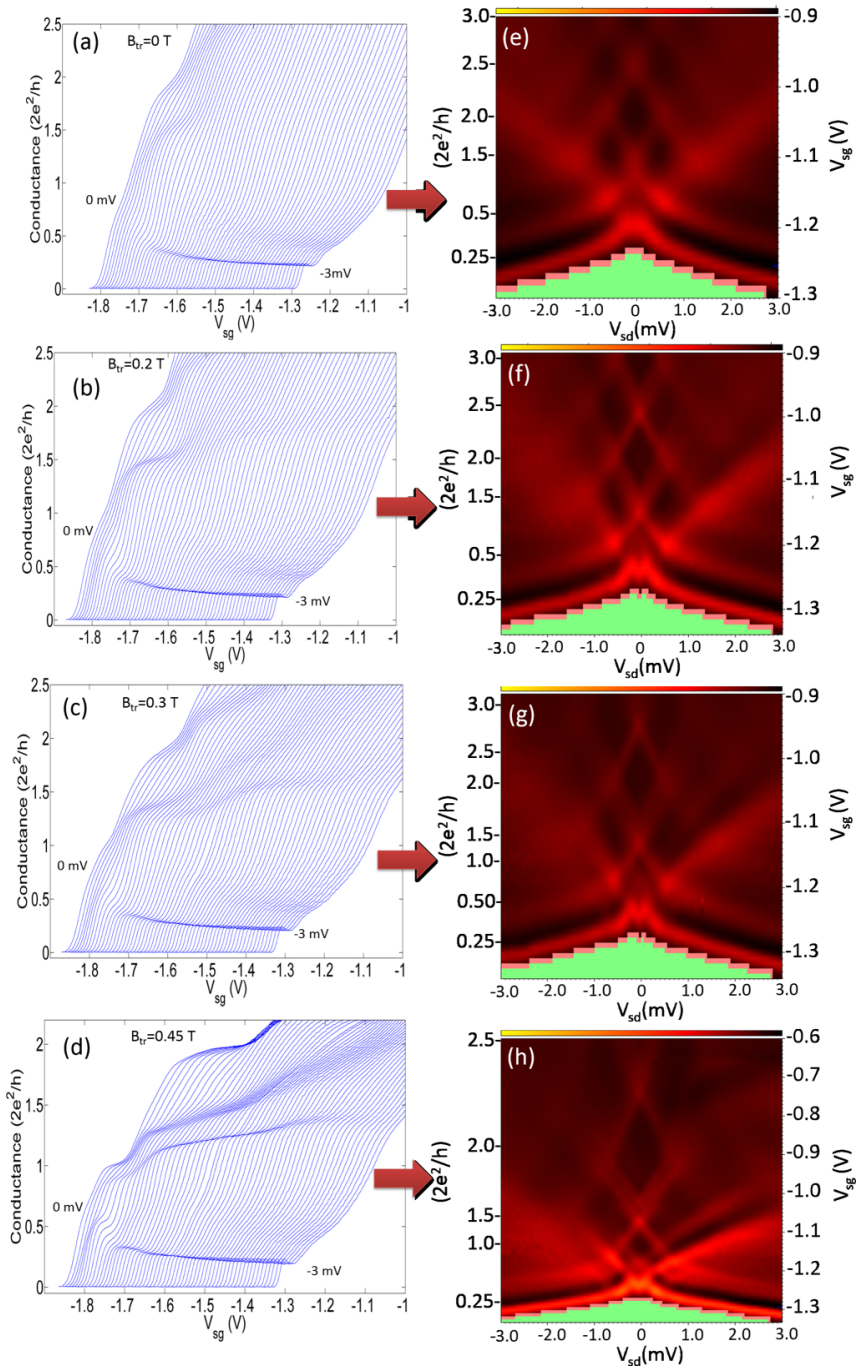

FIG. 2. (Color online) dc-bias characteristics measured in the presence of $B_{\text {tr }}$. (a)-(d) show plots of conductance characteristics for a source-drain bias of 0 to $-3 \mathrm{mV}$ in the presence of $B_{\text {tr }}$ of $0,0.2$, 0.3 , and $0.45 \mathrm{~T}$, respectively. The traces have been offset horizontally for clarity. The trace on the extreme left (right) corresponds to $0 \mathrm{~V}$ $(-3 \mathrm{mV}) \mathrm{dc}$ bias, and the successive traces are taken at intervals of $0.5 \mathrm{mV}$. (e)-(h) represent grayscale plots of $d G / d V_{\mathrm{sg}}$ for the data in (a)-(d), respectively; the red regions are the conductance risers and black regions are conductance plateaus.

the 1-state, giving rise to an anticrossing and a jump to $4 e^{2} / h$, as indicated in the blue trace at around $V_{\mathrm{tg}}=-8.53 \mathrm{~V}$ in Fig. 3(a) [see also Fig. 3(c)]. It may be noted that a number of traces in close proximity to the blue trace exhibited direct jumps to $4 e^{2} / h$, which indicates that the two rows are stable in this regime. We note that as there is only one conductance plateau, the two levels formed by the hybridization are degenerate, i.e., the two rows. The difference in the rate of movement of the two levels illustrates that their energies are not just determined by spatial confinement, which would cause them to move in a similar manner. The energy of the 0-state is more insensitive to the confinement, and after crossing the 1 -state continues to rise relative to the other higher states [Fig. 3(c)]. This feature is also indicated by the
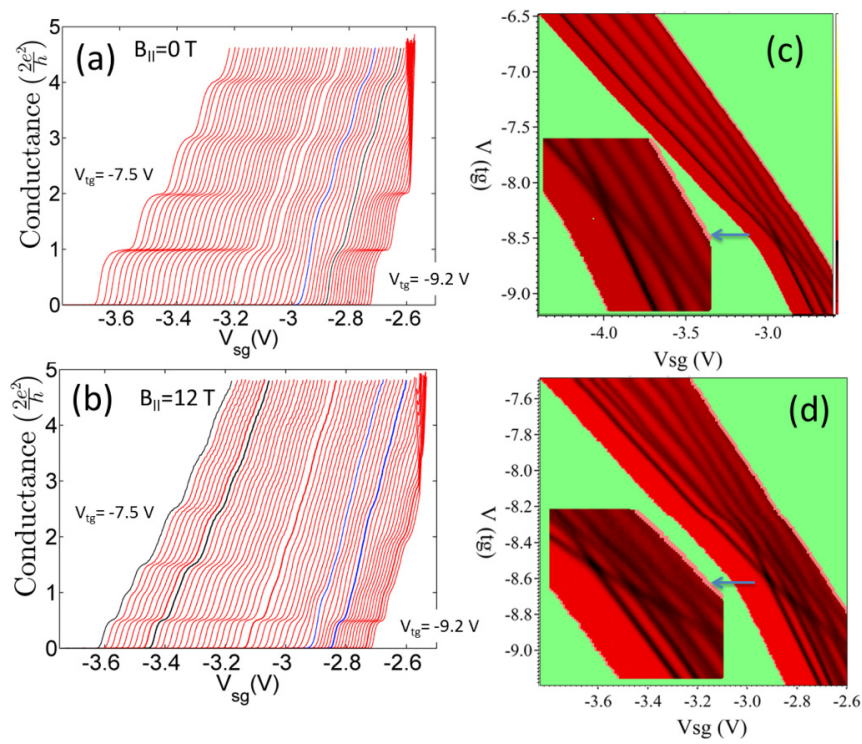

FIG. 3. (Color online) (a) Conductance characteristics of the device in a different cooldown for different $V_{\mathrm{tg}}(-7.5$ to $-9.2 \mathrm{~V})$. As the confinement weakens, the plateaus corresponding to $2 e^{2} / h$ and $4 e^{2} / h$ smear out and then reappear. (b) Conductance characteristics in the presence of $B_{\|}$of $12 \mathrm{~T}$ showing Zeeman splitting and crossing of the 0 -state with the higher subbands as the confinement weakens. (c) and (d) show $d G / d V_{\mathrm{sg}}$ plots represented as grayscale for the data in (a) and (b), respectively, where dark regions represent the conductance risers and the red regions are the conductance plateaus. The insets of (c) and (d), indicated by arrows, show the magnified view of anticrossing and crossing, respectively, of the 0 -state with the 1 and other higher states.

closer proximity of the conductance plots to each other as a function of split-gate voltage occurring when the 1-state drops to become the new ground state [see traces on the right of the green trace in Fig. 3(a)]. If the effect was purely electrostatic, due to the dependence of channel formation on the top- and split-gate voltages, then a gradual transition may be expected. The sharpness of the transition indicates that the ground state formed by the 1-state is less dependent on the carrier concentration, and the electron-electron interaction, than that when the 0 -state was the ground state. As the confinement is further weakened, when $V_{\mathrm{tg}} \leqslant-8.75 \mathrm{~V}$, the 0 -state rises above the 1-state and anticrosses the 2 -state, giving a jump to $6 e^{2} / h$ as there is no longer a separate 2-state, as shown by the green trace in Fig. 3(a). This effect consequently occurs only when the $2 e^{2} / h$ is reintroduced as the first plateau, due to the 1-state being the new ground state. An important aspect of this effect is that jumps due to the omission of higher plateaus are only observed when the 0 -state is passing up through the levels and consequently the first $2 e^{2} / h$ is present. The return of the $4 e^{2} / h$ plateau is accompanied by a tendency for the $6 e^{2} / h$ to disappear with a jump from $4 e^{2} / h$ to $8 e^{2} / h$, however, the grayscale plot is not sufficiently clear for the cause to be established unambiguously.

The role of the $12 \mathrm{~T}$ in-plane magnetic field is immediately apparent by the overall increased closeness of the conductance plots in Fig. 3(b) arising from the lifting of the spin degeneracy. This is clearest for strong confinement where the conductance is given by $N e^{2} / h$, where $N$ is $1,2,3,5 \ldots$ The missing 4 
and 6 arise from overlap of spin down and spin up levels from different subbands [see the first black trace in Fig. 3(b)]. Assuming the spin down $\downarrow$ state to be the lowest spin energy, and adopting the same indexation of states as previously, we see from an inspection of Fig. 3(b) that a merger of the $1 \uparrow$ and $2 \downarrow$ states occurs, and that as the channel is widened, they stay together. In a similar vein, as the channel widens, the overlap of the $0 \uparrow$ and $1 \downarrow$ states takes place and in the conductance plot the plateau with an integer value $N=2$ disappears [the dark, black trace near $V_{\mathrm{sg}}=-3.4 \mathrm{~V}$ in Fig. 3(b)]. As the channel widens, they do not diverge, but the levels have formed a stable state; this may be related to previous observations of energy level locking in parallel quantum wires [24,25]. Possibly this effect is enhanced by the opposite spin polarizations of the two levels participating in the locking.

Widening the channel further results in a complex pattern of overlapping levels, which is similar to another example of level hybridization due to quasi-1D channels formed in coupled electron gases [26]. When the $V_{\text {sg }}$ threshold is approximately $-3.13 \mathrm{~V}$, a gap appears in the traces and an integer value of $N=4$ appears, although $N=2$ continues to be absent; from then on $N=3$ is very weak and disappears. The gap in the characteristics, particularly at $V_{\mathrm{sg}} / V_{\mathrm{tg}}=-3.13 \mathrm{~V} /-8.26 \mathrm{~V}$ (the dark-red trace), is an indicator of an increased density of states as the levels overlap. A spin-polarized state of the double row is at $2 e^{2} / h$ for $V_{\mathrm{sg}} / V_{\mathrm{tg}}=-2.95 \mathrm{~V} /-8.6 \mathrm{~V}$, as indicated by the light-blue trace. The stability of the system is shown by the observation of a jump from $0.5 e^{2} / h$ to $2.5 e^{2} / h$, indicated by the dark-blue trace at $V_{\mathrm{sg}} / V_{\mathrm{tg}}=-2.75 \mathrm{~V} /-8.8 \mathrm{~V}$, which is a spin-polarized form of the jump $\left(2 e^{2} / h\right.$ to $\left.6 e^{2} / h\right)$ observed in the absence of the magnetic field, indicated by the green trace in Fig. 3(a).

Insight into the processes causing such behavior can be drawn from the grayscale plot in Fig. 3(d), where we see that the ground $0 \downarrow$ changes at a much slower rate than the higher levels, so cutting across them and giving rise to the pattern of crossing events. This is similar to the zero magnetic field case but greatly enhanced, so accounting for the reduced region of row formation where the $e^{2} / h$ disappears and the first plateau is $2 e^{2} / h$. The increasing hybridization as levels move together also explains why a new plateau emerges from one plateau then drops in value before settling at the next quantized value [27]. A significant feature of the conductance plots is the disappearance of the $2 e^{2} / h$ plateau and the reintroduction of $e^{2} / h$ as the ground state; this arises from the $2 \downarrow$ dropping through many states to become the new ground state. This is a surprising manifestation of the modification of the levels by the electron interaction. The 0 -state passes through several levels during its trajectory, but the results are not sufficiently clear to determine the spin dependence of hybridization.

In conclusion, we have studied electron transport in a quantum wire where the confinement is altered by a combination of split gates and a top gate. When the confinement is weakened, the conductance plateau at $2 e^{2} / h$ disappears, whereas the $4 e^{2} / h$ plateau persists and becomes the new ground state comprising two rows. Monitoring the movement of energy levels, it appears that the electrons in the ground state (0-state) hybridize with the first excited state (1-state) to give rise to $4 e^{2} / h$ as a first plateau, and then a further reduction of the confinement results in the 1 -state falling below the 0 -state, and a $2 e^{2} / h$ plateau reappears. It has been found that the electronelectron interaction affects the ground state more significantly than the higher levels, causing it to move through them as the confinement weakens, so modifying the sequence of quantized plateaus. Such movement of energy levels as a consequence of a Coulomb interaction gives further insight on the conductance behavior of weakly confined quasi-1D systems.

We thank Dr. J. T. Nicholls and Professor G. Gumbs for many fruitful discussions. This work was supported by the Engineering and Physical Sciences Research Council (EPSRC), UK.
[1] T. J. Thornton, M. Pepper, H. Ahmed, D. Andrews, and G. J. Davies, Phys. Rev. Lett. 56, 1198 (1986).

[2] K.-F. Berggren, T. J. Thornton, D. J. Newson, and M. Pepper, Phys. Rev. Lett. 57, 1769 (1986).

[3] D. A. Wharam, T. J. Thornton, R. Newbury, M. Pepper, H. Ahmed, J. E. F. Frost, D. G. Hasko, D. C. Peacock, D. A. Ritchie, and G. A. C. Jones, J. Phys. C 21, L209 (1988).

[4] B. J. van Wees, H. van Houten, C. W. J. Beenakker, J. G. Williamson, L. P. Kouwenhoven, D. van der Marel, and C. T. Foxon, Phys. Rev. Lett. 60, 848 (1988).

[5] Y. Jompol, C. Ford, J. Griffiths, I. Farrer, G. Jones, D. Anderson, D. Ritchie, T. Silk, and A. Schofield, Science 325, 597 (2009).

[6] K. J. Thomas, J. T. Nicholls, M. Pepper, W. R. Tribe, M. Y. Simmons, and D. A. Ritchie, Phys. Rev. B 61, R13365(R) (2000); K. J. Thomas, J. T. Nicholls, N. J. Appleyard, M. Y. Simmons, M. Pepper, D. R. Mace, W. R. Tribe, and D. A. Ritchie, 58, 4846 (1998).

[7] T.-M. Chen, A. C. Graham, M. Pepper, F. Sfigakis, I. Farrer, and D. A. Ritchie, Phys. Rev. B 79, 081301(R) (2009).
[8] F. Sfigakis, C. J. B. Ford, M. Pepper, M. Kataoka, D. A. Ritchie, and M. Y. Simmons, Phys. Rev. Lett. 100, 026807 (2008).

[9] W. K. Hew, K. J. Thomas, M. Pepper, I. Farrer, D. Anderson, G. A. C. Jones, and D. A. Ritchie, Phys. Rev. Lett. 102, 056804 (2009).

[10] L. W. Smith, W. K. Hew, K. J. Thomas, M. Pepper, I. Farrer, D. Anderson, G. A. C. Jones, and D. A. Ritchie, Phys. Rev. B 80, 041306(R) (2009).

[11] P. M. Wu, P. Li, H. Zhang, and A. M. Chang, Phys. Rev. B 85 , 085305 (2012).

[12] J. S. Meyer and K. Matveev, J. Phys.: Condens. Matter 21, 023203 (2009).

[13] A. D. Klironomos, J. S. Meyer, T. Hikihara, and K. A. Matveev, Phys. Rev. B 76, 075302 (2007).

[14] H. Lind, I. I. Yakimenko, and K.-F. Berggren, Phys. Rev. B 83, 075308 (2011).

[15] E. Welander, I. I. Yakimenko, and K.-F. Berggren, Phys. Rev. B 82, 073307 (2010).

[16] G. W. Bryant, Phys. Rev. Lett. 59, 1140 (1987). 
[17] F. Bloch, Z. Phys. 57, 545 (1929).

[18] G. Pagano, M. Mancini, G. Cappellini, P. Lombardi, F. Schäfer, H. Hu, X.-J. Liu, J. Catani, C. Sias, M. Inguscio, and L. Fallani, Nat. Phys. 10, 198 (2014).

[19] In this configuration the carrier concentration can be manipulated by adjusting the top gate and the split gate voltages so that the confinement potential is weakened, allowing the electrons to relax in the direction normal to the direction of current.

[20] Referring to Fig. 2(e), it may be noted that a strong crossing is seen near $1.5\left(2 e^{2} / h\right)$, which continues across the higher subbands as the source-drain voltage is increased. The sourcedrain voltage where this crosses $1.5\left(2 e^{2} / h\right)$ is also the value corresponding to the appearance of the $0.5\left(2 e^{2} / h\right)$ structure.

[21] The plateaus are displaced from the standard values, probably due to a series resistance created by the transverse magnetic field. Also, the series resistance has not been removed for the grayscale plots in Fig. 2 and Fig. 3.
[22] H. van Houten, C. W. J. Beenakker, P. H. M. van Loosdrecht, T. J. Thornton, H. Ahmed, M. Pepper, C. T. Foxon, and J. J. Harris, Phys. Rev. B 37, 8534(R) (1988).

[23] We have observed the double jump with a number of devices, and at least on ten occasions for different cooldowns. We demonstrate that the characteristics are very smooth with no fluctuations, a necessary condition for having confidence in the absence of defects or impurity aided effects.

[24] C. G. Smith, M. Pepper, R. Newbury, H. Ahmed, D. G. Hasko, D. C. Peacock, J. E. F. Frost, D. A. Ritchie, G. A. C. Jones, and G. Hill, J. Phys.: Condens. Matter 1, 6763 (1989).

[25] Y. Sun and G. Kirczenow, Phys. Rev. Lett. 72, 2450 (1994).

[26] K. J. Thomas, J. T. Nicholls, M. Y. Simmons, W. R. Tribe, A. G. Davies, and M. Pepper, Phys. Rev. B 59, 12252 (1999).

[27] A. C. Graham, K. J. Thomas, M. Pepper, N. R. Cooper, M. Y. Simmons, and D. A. Ritchie, Phys. Rev. Lett. 91, 136404 (2003). 